\title{
The role of cerebral amyloid $\beta$ accumulation in common forms of Alzheimer disease
}

\author{
Sam Gandy \\ Farber Institute for Neurosciences, Thomas Jefferson University, Philadelphia, Pennsylvania, USA.
}

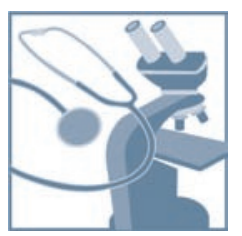

For approximately 80 years following Alzheimer's description of the disease that bears his name, a gulf divided researchers who believed that extracellular deposits of the amyloid $\beta(A \beta)$ peptide were pathogenic from those who believed that the deposits were secondary detritus. Since 1990, the discoveries of missense mutations in the A $\beta$ peptide precursor (APP) and the APP-cleaving enzyme presenilin 1 (PS1) have enabled much progress in understanding the molecular, cellular, and tissue pathology of the aggregates that accumulate in the interstices of the brains of patients with autosomal dominant familial Alzheimer disease (AD). Clarification of the molecular basis of common forms of $\mathrm{AD}$ has been more elusive. The central questions in common $\mathrm{AD}$ focus on whether cerebral and cerebrovascular $A \beta$ accumulation is (a) a final neurotoxic pathway, common to all forms of $A D$; (b) a toxic by-product of an independent primary metabolic lesion that, by itself, is also neurotoxic; or (c) an inert by-product of an independent primary neurotoxic reaction. Antiamyloid medications are entering clinical trials so that researchers can evaluate whether abolition of cerebral amyloidosis can mitigate, treat, or prevent the dementia associated with common forms of $\mathrm{AD}$. Successful development of antiamyloid medications is critical for elucidating the role of $A \beta$ in common $A D$.

Alzheimer disease (AD) is the most common cause of dementia, a clinicopathological state whose name literally means "loss of the ability to think." There is much disagreement even among AD specialists about the basic nature of the disease, and that controversy is no better illustrated than in contemplation of how the disease is initiated at the molecular level. Histologically, the neuronal cytoskeleton twists, literally, into structures called neurofibrillary tangles (NFTs). Outside the cell, the amyloid $\beta(A \beta)$ peptide aggregates into clumps called oligomers, which accumulate and form deposits called amyloid plaques. Based on studies of a syndrome known as mild cognitive impairment (MCI) (a possible prodrome to dementia), the development of detectable entorhinal NFTs is considered to be the histological correlate of MCI and, many believe, the harbinger of incipient AD (1). Still, levels of cortical synaptic markers correlate with cognitive status at time of death better than do either plaque load or tangle load (2), which is consistent with the concept that neurotransmission failure is the proximate cause of cognitive decline.

Amyloid is a highly ordered precipitate of extracellular protein, misnamed "starch-like" by Rudolf Virchow because of its reactivity to the PAS stain (3). Systemic amyloid deposits can occur in any organ and are often large and amorphous; cerebral amyloid deposits take the form of delimited, miliary spheres called plaques.

Nonstandard abbreviations used: $A \beta$, amyloid $\beta$; $A B$ ri, British amyloid; $A D$, Alzheimer disease; ADAM, a disintegrin and metalloproteinase; ADan, Danish amyloid; ADDL, A $\beta$-derived diffusible ligand; AICD, APP intracellular domain; APP, $\mathrm{A} \beta$ peptide precursor; BACE, $\beta$-APP site-cleaving enzyme; C89, $\beta^{\prime}$-cleaved carboxyterminal fragment of APP; CTF $\beta$, $\beta$-cleaved carboxyterminal fragment of APP (also known as C99); FBD, familial British dementia; FDD, familial Danish dementia; FTD, frontotemporal dementia; HRT, hormone replacement therapy; MCI, mild cognitive impairment; NFT, neurofibrillary tangle; PF, protofibril; PM, plasma membrane; PP, protein phosphatase; PS, presenilin; ROCK, rho-associated protein kinase; $\mathrm{SAPP}_{\alpha}$, soluble $\mathrm{APP}_{\alpha}$.

Conflict of interest: The author currently receives grant support from the Robert $\mathrm{C}$ Atkins Foundation and Neurochem Inc. and consultant fees from representatives of the Women's Health Research Institute of Wyeth Inc. and Elan Pharmaceuticals Inc.

Citation for this article: J. Clin. Invest. 115:1121-1129 (2005).

doi:10.1172/JCI200525100.
Plaques contain a trace amount of glycosaminoglycans, which explains the PAS positivity. To the neuropathologist, the diagnosis of "amyloid" is applied to any proteinaceous tissue precipitate that binds the dye Congo red. Congophilia is a property of all amyloids and is related to the defining ability of these precipitates to form $\beta$-pleated sheets that subsequently assemble into fibrils (4).

In $\mathrm{AD}$, brain amyloid is composed almost entirely of a $4 \mathrm{kDa}$ amyloid $\beta(\mathrm{A} \beta$ ) peptide (5) that exhibits microheterogeneity in amino acid sequence and in a variety of biophysical states (Figure 1). Most $A \beta$ is comprised of a peptide designated $A \beta 40, A \beta_{40}, A \beta_{1-40}$, or, in some cases, $A \beta_{x-40}$. Peptides with various amino termini, all bearing an identical carboxyl terminus, form a major proportion (greater than $95 \%$ ) of the total $A \beta$ produced by cells (6). A minor fraction (less than $5 \%$ ) of the newly generated $A \beta$ ends at residue 42 (6). This "long $A \beta$ " (also abbreviated as $A \beta_{1-42}, A \beta 42, A \beta_{42}$, or $A \beta_{x-42}$, the latter 3 representing species with heterogeneous amino termini) is much more aggregatable than $A \beta_{40}$; hence, "long $A \beta$ " is believed to initiate the formation of oligomers, fibrils, and plaques (7).

The generation of $A \beta$ from its precursor, the $A \beta$ peptide precursor (APP), is illustrated in Figure 2. APP is first cleaved at the amino terminus of $A \beta$ by a membrane-bound aspartyl protease ( $\beta$-secretase). This cleavage generates a large secreted derivative (soluble $\mathrm{APP}_{\beta}\left[\mathrm{sAPP}_{\beta}\right]$ ) and a membrane-bound $\beta$-cleaved carboxyterminal fragment of APP (CTF $\beta$; also known as C99). Cleavage of CTF $\beta$ by $\gamma$-secretase results in the production of the $A \beta 40$ and $A \beta 42$ species described above. The term "soluble $A \beta$ " generally is applied either to newly generated, cell-secreted $A \beta$ or to that fraction of tissue or synthetic $A \beta$ that is taken into the aqueous phase of a non-detergent-containing extraction buffer. "Misfolded" and "aggregated" $\mathrm{A} \beta$ are terms used to describe very early, nonspecific changes in $A \beta$ folding states or solubility states, respectively (e.g., aggregated A $\beta$ solutions usually scatter light to a greater extent than do solutions of soluble $A \beta)$. "Oligomeric" A $\beta$ refers to peptide assemblies with limited stoichiometry (e.g., dimers, trimers, etc.), while protofibrils (PFs) are structures of intermediate order between aggregates 
A

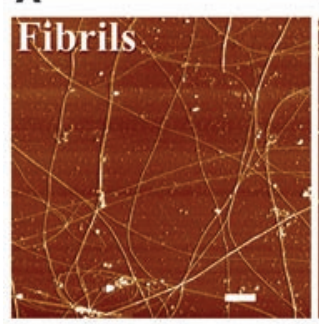

B

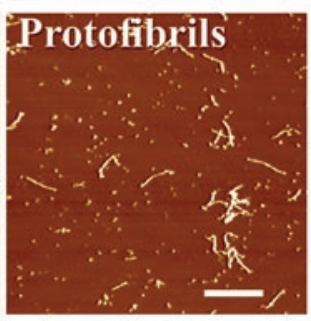

c

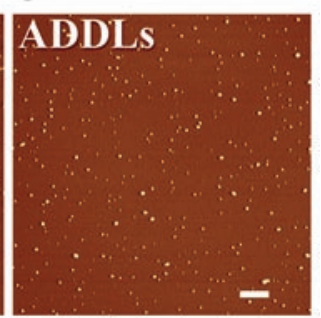

Figure 1

Different assembly (biophysical) states of $A \beta$. The assembled forms obtained from incubation of synthetic $A \beta$ are highly sensitive to preparation and incubation. Widely differing proportions of insoluble fibrils (A), soluble PFs (B), and oligomers (also known as ADDLs) are revealed by atomic force microscopy. Typical PF and fibril preparations contain varying levels of small globular molecules, putatively $A \beta$ oligomers (ADDLs). ADDL preparations (C) initiated from monomeric dimethyl sulfoxide stock solutions are fibril- and PFfree and uniquely comprise oligomers. Scale bars: $200 \mathrm{~nm}$. Figure reproduced with permission from Trends in Neurosciences (8). and fibrils. The term "A $\beta$-derived diffusible ligands" (ADDLs) is also applied to pre-protofibrillar intermediates (Figure 1), based less on a structural definition than on the neurotoxic activity of these oligomers. Indeed, oligomers, PFs, and ADDLs are believed to be the assembly states of $A \beta$ with the most potent toxicity and are believed by many in the field to be the proximate mediators of $A \beta$-induced neurotoxicity, especially in primary neuronal culture models $(8,9)$. The final assemblies, called fibrils, are the basic building blocks of the amyloid plaque (Figure 3 ) and are so named because of their characteristic ultrastructural appearance.
Amyloids exhibit a typical exponential growth property known as seeding, which means that once a few fibrils are formed, they instruct the misfolding of other amyloidogenic peptides. The transmissibility of another amyloidosis, prion disease, can be viewed as an extreme example of seeding. In that case, the activation energy and favorability of alternative, neurotoxic forms of the prion protein are believed to permit aggressive propagation of fibrillogenesis. Of note, prion diseases, such as Creutzfeldt-Jakob disease, provide the clearest heuristic evidence that amyloids can be neurotoxic and that amyloid plaques per se are not required for neurotoxicity and clinical disease (10).

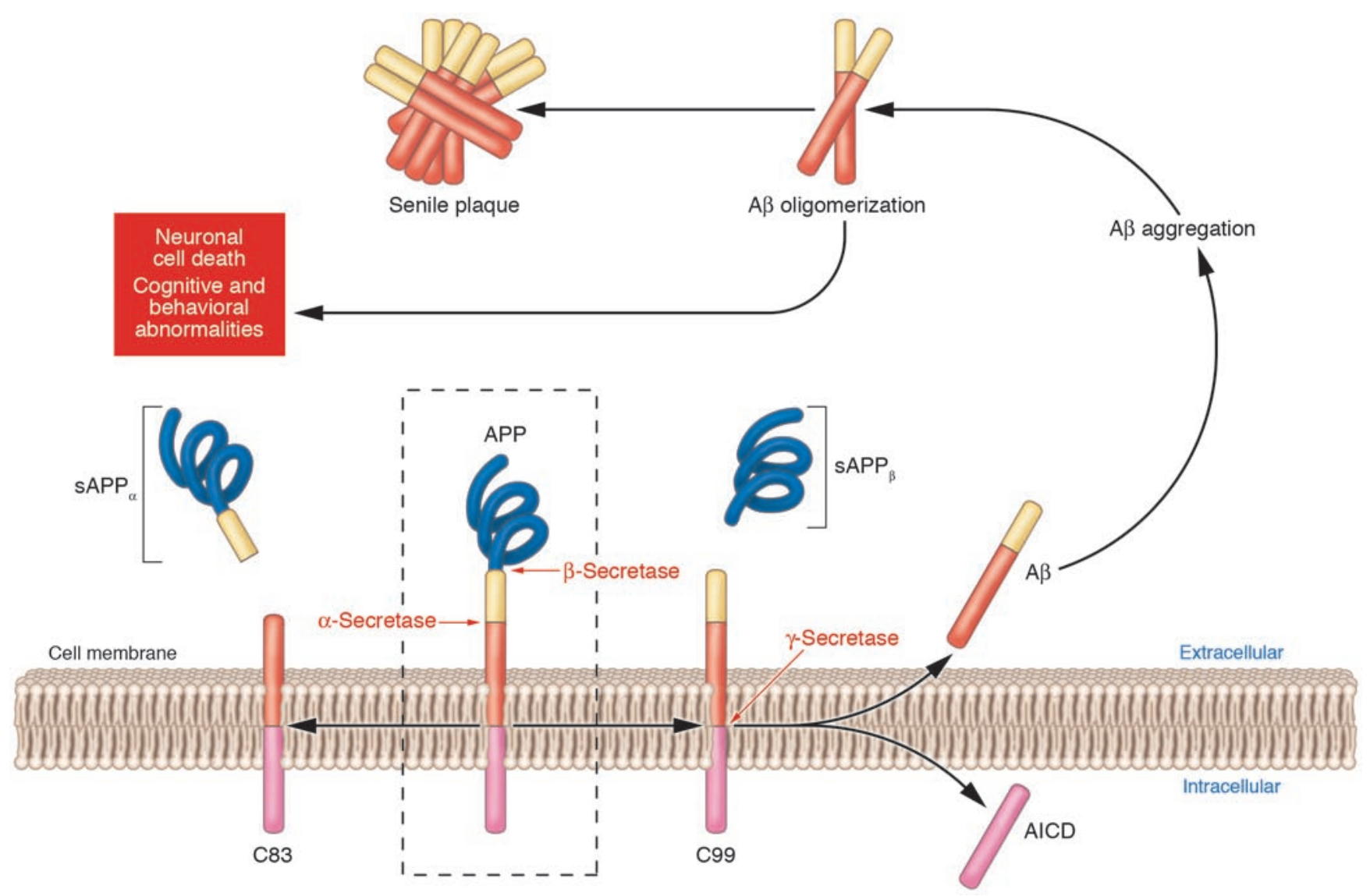

Figure 2

APP processing and A $\beta$ accumulation. Mature APP (center, inside dashed box) is metabolized by 2 competing pathways, the $\alpha$-secretase pathway that generates SAPP $\alpha$ and C83 (also known as CTF $\alpha$; left) and the $\beta$-secretase pathway that generates sAPP snd $_{3}$ a9 (right). Some $\beta$-secretase cleavage is displaced by 10 amino acid residues and generates sAPP $\beta^{\prime}$ and C89 (see Figure 4). All carboxyterminal fragments (C83, C99, and C89) are substrates for $\gamma$-secretase, generating the APP intracellular domain (AICD) and, respectively, the secreted peptides p3 (not shown), $A \beta$ (right), and Glu ${ }^{11} A \beta$ (see Figure 4). A $\beta$ aggregates into small multimers (dimers, trimers, etc.) known as oligomers. Oligomers appear to be the most potent neurotoxins, while the end stage senile plaque is relatively inert. 


\section{What is the relationship between amyloidosis and cognitive decline?}

Neuropathologists have long recognized that - among the various structural markers of $\mathrm{AD}$ - plaque burden is the poorest correlate of cognitive status at the time of death. As more refined techniques for measuring $A \beta$ levels have become available (e.g., ELISA), this correlation has been revisited again and again. Early ELISA correlations between brain $A \beta$ levels and cognitive status were not much better than the histological correlation of cognitive status and plaque load, due, at least in part, to the seeding phenomenon: once fibrils and plaques begin to form, their concentrations rise rapidly, over several orders of magnitude. The massive levels of plaque $A \beta$ achieved by exponential fibrillogenesis are not readily quantitatively solubilizable, and the interindividual differences in solubilizable $A \beta$ are believed to be a source of much variability and noise in studies aimed at determining clinicopathological correlation between amyloid load and cognitive status, since small amounts of differences in amyloid solubilizabililty can cause dramatic variability in A $\beta$ levels measured by ELISA. More recently, Naslund et al. revisited the correlation between $A \beta$ and cognitive status using brains from well-characterized subjects at the threshold between MCI and dementia, where contamination by plaque $A \beta$ is least problematic (11). In this study, for the first time, a strong correlation between $\mathrm{A} \beta$ concentration and cognitive status was documented. These results dovetail well with data obtained using transgenic mice that possess a mutant form of APP (Tg2576 mice), which show that memory deficits correlate with $\mathrm{A} \beta$ elevation and precede plaque formation (12). More recently, brain and cerebrospinal fluid levels of $A \beta$ oligomers (ADDLs) have been reported to correlate closely with cognitive status $(13,14)$. Taken together, these 3 studies (11-14) go a long way toward resolving the heretofore apparently poor correlation between cognition and either $\mathrm{A} \beta$ levels or amyloid load.

\section{What is the strongest evidence that AD can ever begin with amyloidosis?}

Genetic evidence links altered $A \beta$ metabolism to the rare subset of $\mathrm{AD}$ that is autosomal dominant and completely penetrant. These forms of $\mathrm{AD}$ share a single common feature, and that is the facilitation of oligomerization of $A \beta$. Usually the phenotype involves an obvious change in the proteolytic processing of APP. Missense mutations within APP are clustered around the $\mathrm{N}$ and $\mathrm{C}$ termini of the $\mathrm{A} \beta$ domain, and the usual phenotype involves increased generation of the hyperaggregatable $A \beta$ species that end(s) at residue 42 (Figure 4; reviewed in ref. 15). Familial AD pedigrees have been identified worldwide, and the geography of the initial discovery is often used to name the mutant molecule (e.g., Sweden, Holland, Britain, Indiana, Flanders, etc). A few mutations occur within the $\mathrm{A} \beta$ domain that result in increased intrinsic aggregatability of all $A \beta$ species. A recently discovered pathogenic mutation, found in $\mathrm{AD}$ patients from northern Sweden, near the Arctic Circle, actually decreases the levels of total $\mathrm{A} \beta$ generation, yet the molecules that are generated have an increased propensity to oligomerize $(16,17)$. Pathogenic APP mutations are the rarest known genetic cause of familial $\mathrm{AD}$, being responsible for disease in only about 50 families worldwide. Still, the existence of familial AD due to proamyloidogenic APP mutations around the A $\beta$ domain and the final common phenotype (promotion of $A \beta$ aggregation) provides the most compelling evidence that the entire clinicopathological picture of $\mathrm{AD}$ can - at least sometimes - begin with $\mathrm{A} \beta$ aggregation. None of

\section{Figure 3}

Amyloid plaque-forming transgenic mice and positron emission tomography (PET) scans of amyloid plaque load in normal human subjects and subjects with AD. (A) Thioflavin staining of subiculum of control mouse, aged 14 months. Fluorescence is nonspecific and cellular. Magnification, $\times 20$. (B) Thioflavin staining of littermate, mutant APP $X$ mutant PS mouse, demonstrating thioflavinpositive amyloid plaques. Magnification, $\times 20$. (C) Immunostaining of amyloid plaque from cortex from same mouse as in B. Magnification, $\times 40$. Figures courtesy of Michelle Ehrlich (Thomas Jefferson University). (D) $\left[{ }^{18} \mathrm{~F}\right] \mathrm{FDDNP}$ PET scan (to examine amyloid plaque and NFT load), MRI, and fluorodeoxy-glucose (FDG) PET (to examine glucose metabolism) images of a subject with $A D$ and a control normal subject. The $\left[{ }^{18} \mathrm{~F}\right] \mathrm{FDDNP}$ and FDG (summed) images are coregistered to their respective MRI images. Areas of FDG hypometabolism (blue) are matched with the localization of amyloid plaques and NFTs as visualized by $\left[{ }^{18} \mathrm{~F}\right]$ FDDNP binding. The color bar represents the scaling of the $\left[{ }^{18} \mathrm{~F}\right] \mathrm{FDDNP}$ and FDG images. FDDNP, 2-(1-[6-[(2$\left[{ }^{18} \mathrm{~F}\right]$ fluoroethyl)(methyl)amino]-2-naphthyl] ethylid ene)malononitrile; max, maximum; min, minimum. Figure reproduced with permission from the American Journal of Geriatric Psychiatry (62).
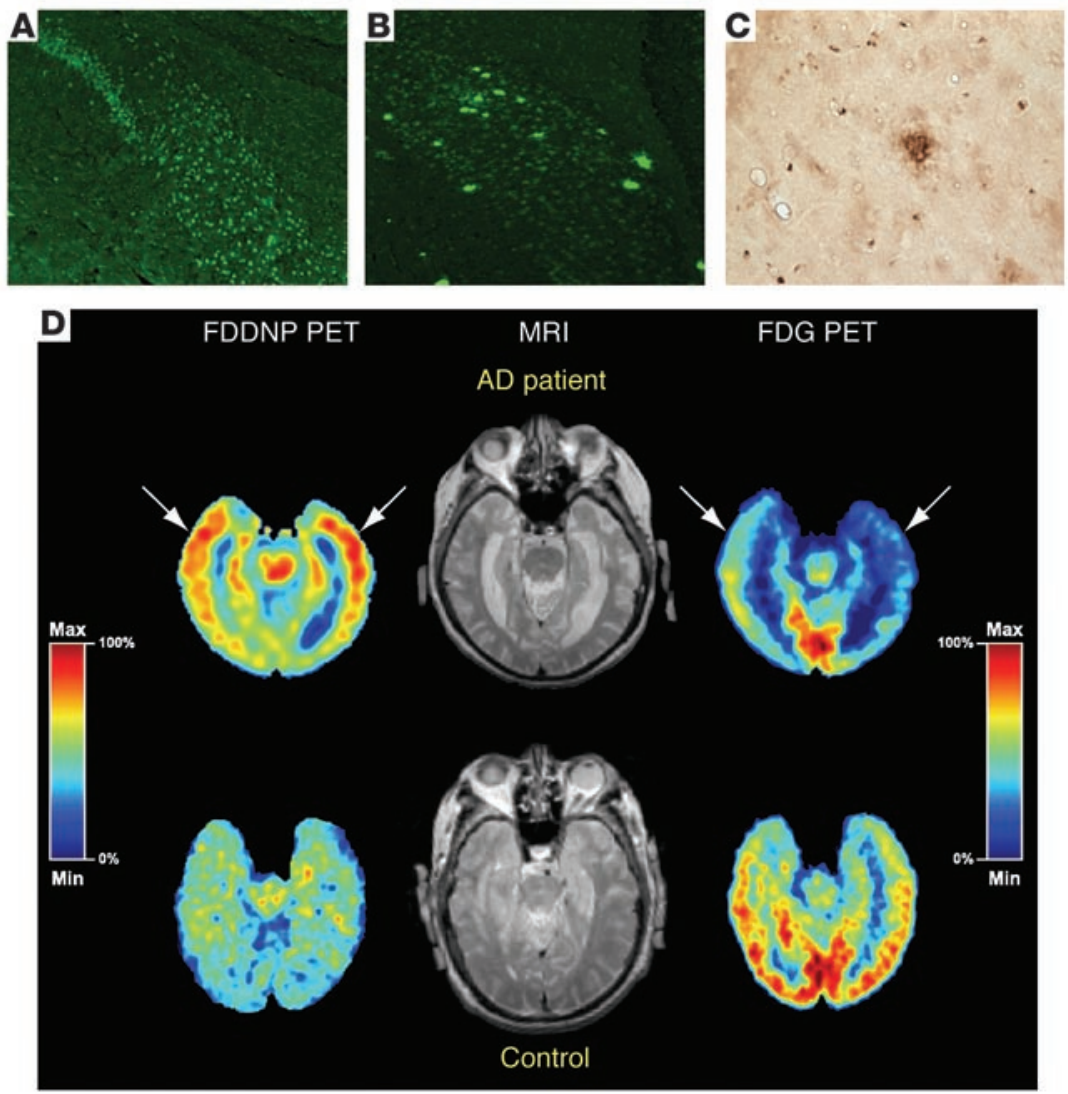


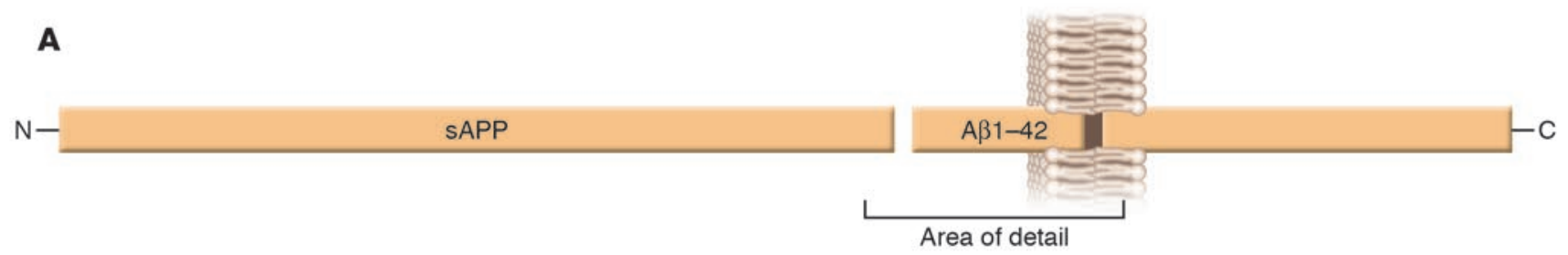

B

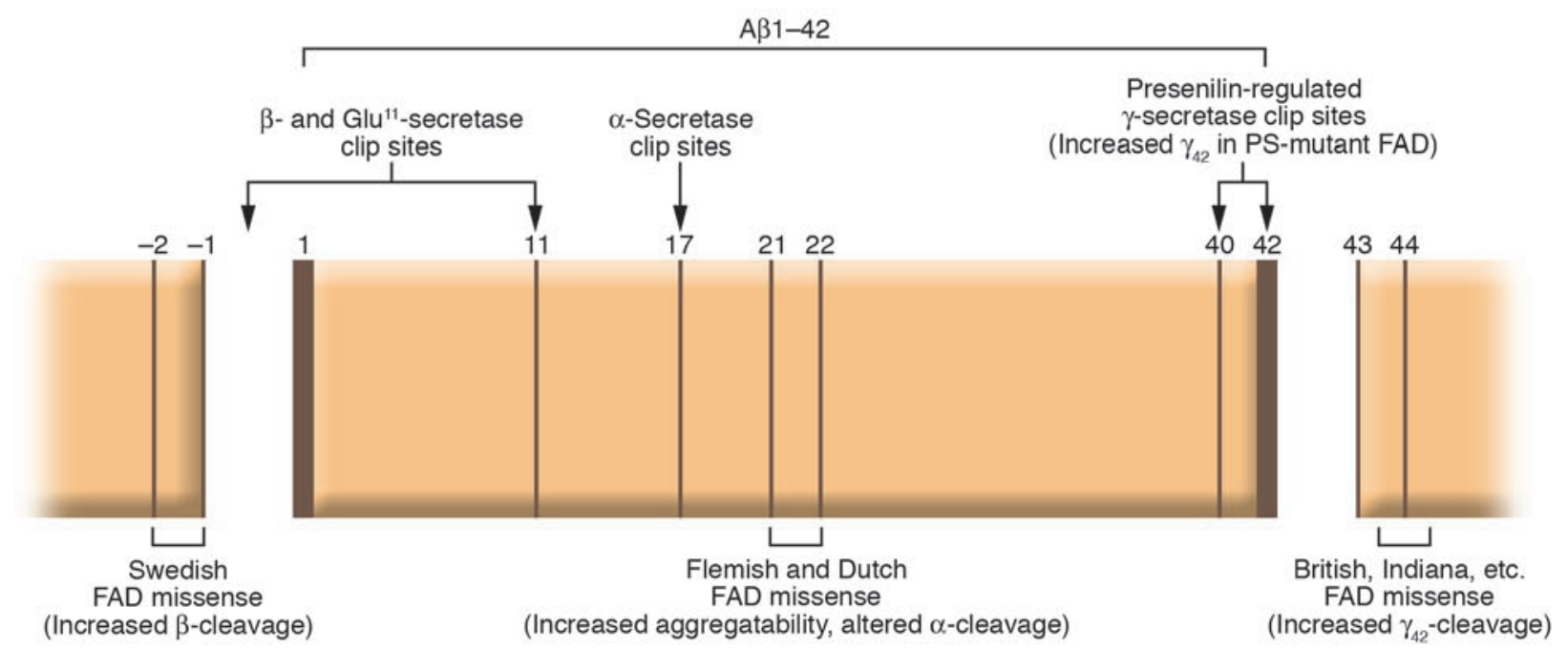

Figure 4

(A) Structure and topology of APP. (B) The fine structure around the A $\beta$ domain, secretase cleavage sites, and locations of some selected familial AD missense mutations. Figure modified with permission from Trends in Endocrinology and Metabolism:TEM (15).

these pathogenic mutations has ever been identified in any individual who failed to develop dementia, with the notable exception of a handful of members of a mutant $A P P$ family who possessed not only a mutant form of $A P P$ but also the $\varepsilon 2$ allele of the $A P O E$ gene (18). APOE polymorphisms are discussed in more detail below, but the rare cases in which both mutant APP and the APOE $\varepsilon 2$ polymorphism coexist in the same individuals provide the best evidence that $A P O E \varepsilon 2$ can protect against the development of AD.

Additional support for the role of amyloid in the process of neurodegeneration comes from 2 newly described hereditary conditions, familial British and Danish dementias (FBD and FDD, respectively), in which patients suffering from either condition present with extensive amyloid deposition in the CNS that is colocalized with NFTs and associated with neurodegeneration. Although the biochemical properties of NFTs in both FBD and FDD are indistinguishable from those found in $\mathrm{AD}$, the amyloid deposits in the former 2 disorders are composed of 2 new mol- ecules, British amyloid (ABri) and Danish amyloid (ADan), which are structurally unrelated to the $A \beta$ associated with AD (19). ABri and $A D a n$ are generated by missense mutations in the stop codons in the BRI and DAN genes and therefore only exist in nature in humans with these genetic disorders. These illnesses caused by generation of otherwise nonexistent proteins also argue against disease models that describe the origin of cerebral amyloidosis as being due to perturbation of the normal function of the respective amyloid precursors, since the gene for the amyloidogenic peptide is only present in affected individuals.

\section{What is the usual cause of autosomal dominant, familial AD?}

The preponderance of the familial AD for which the defect is known is attributable to completely penetrant missense mutations in the catalytic subunit of $\gamma$-secretase. The mutation is in a complex, 8-transmembrane-domain protein called presenilin (PS) that

\section{Figure 5}

Topology of the 4 components that comprise the high molecular weight $\gamma$-secretase complex. Black bar represents the cleavage site for processing of the zymogen form of PS1 into the amino and carboxyterminal fragments that self associate and form the active enzyme. Figure modified from an image courtesy of Jan Naslund (Karolinska Institute, Stockholm, Sweden).

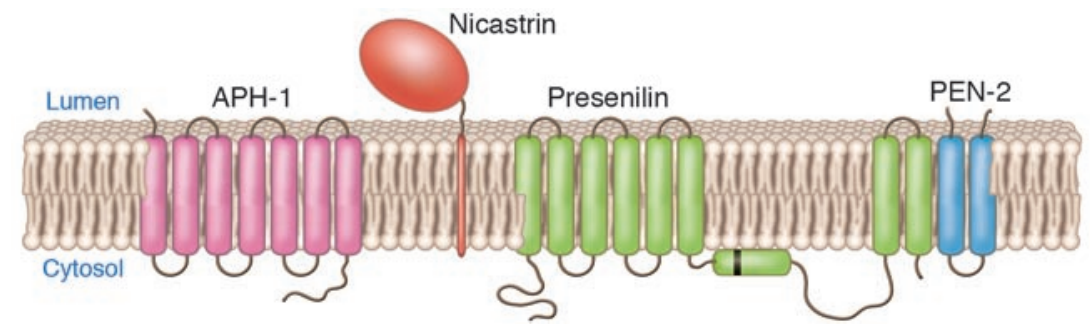




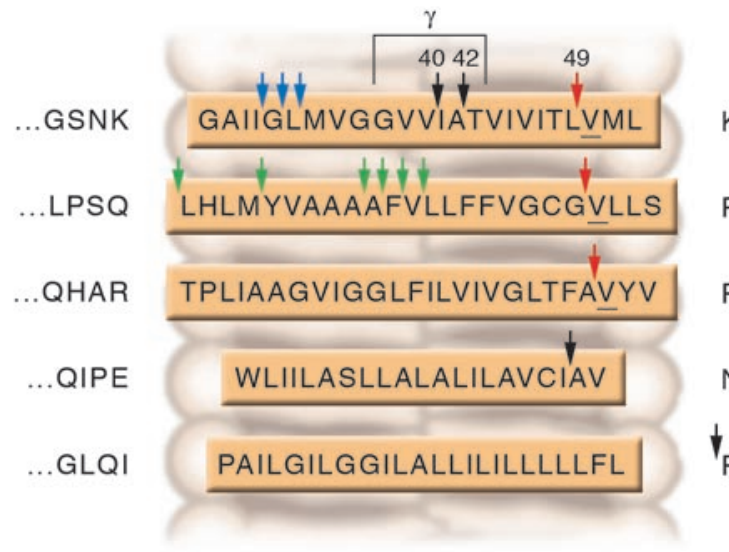

KKKQ... APP

RKRR... Notch1

RRKS... ErbB4

NSRR... CD44

RRRA... E-cadherin

\section{Figure 6}

Intramembranous PS cleavage sites on 5 representative $\gamma$-secretase substrates. Figure modified with permission from Nature Reviews Molecular Cell Biology (61).

cell-associated fragment C83, which is subsequently cleaved by $\gamma$-secretase to yield an $\mathrm{A} \beta$ derivative known as $\mathrm{p} 3$ with an amino terminus beginning at $\mathrm{Leu}^{17}$ of $\mathrm{A} \beta$.

The location of $\alpha$-secretase at the cell surface and $\beta$-secretase in the intracellular and endocytic pathway provides for competition between and mutual exclusivity of the 2 pathways. The $\alpha$-secretase pathway is sensitive to PKC (23), MAPK/ERK (24), rho-associated protein kinase (ROCK) (ref. 25), and

associates with other components known as nicastrin, APH-1, and PEN-2 to form the high molecular weight $\left(>10^{6} \mathrm{kDa}\right.$; see Figure 5) active $\gamma$-secretase that cleaves APP fragments CTF $\alpha$ (the $\alpha$-cleaved carboxyterminal fragment of APP, also known as C83) and CTF $\beta$ and CTF $\beta^{\prime}$ (reviewed in ref. 20).

As with most APP mutations, the mutations in PS appear to act by increasing the generation of the $A \beta 42$ species. However, unlike the relatively simple case of APP mutations (most of which are clustered around the carboxyl terminus of the $A \beta$ domain and favor generation of $A \beta 42$ ), over 150 pathogenic PS mutations, scattered throughout the PS molecule, have been described. A dozen more substrates, in addition to APP, exist for $\gamma$-secretase (Figure 6), and even within APP, $\gamma$-secretase can cleave at several sites, including a site distinct from the canonical $\gamma$-secretase site. Cleavage at this second, so-called " $\varepsilon$-site" liberates the cytoplasmic APP intracellular domain (AICD), which, in analogy with other PS substrates, may traffic to the nucleus and act as a transcription factor (reviewed in ref. 20).

In addition to $\gamma$-secretase, which contains mutations that can cause AD, 2 other secretases control the cleavages that initiate APP processing. An integral aspartyl proteinase, known as $\beta$-secretase or $\beta$-APP-site cleaving enzyme (BACE; ref. 21 ), generates APP carboxyl fragments known as C99 (or CTF $\beta$ ) and C89 ( $\beta^{\prime}$-cleaved carboxyterminal fragment of APP; also known as $C T F \beta^{\prime}$ ), which bear either $A \beta\left[\mathrm{Asp}^{1}\right]$ or $\mathrm{A} \beta\left[\mathrm{Glu}^{11}\right]$ at their amino termini (see Figure $4 \mathrm{~B})$. These cleavage sites are sometimes known as $\beta$ and $\beta^{\prime}$ sites, respectively, and may be differentially generated in endosomes and the trans-Golgi network, respectively. The large ectodomain fragment generated by BACE-mediated cleavage is $\mathrm{SAPP}_{\beta}$. APP is one of only 2 known BACE substrates, which makes BACE inhibition a popular anti-A $\beta$ therapeutic strategy, and the development of safe, orally active BACE inhibitors is being aggressively pursued. To date, success has been limited; the BACE catalytic pocket is especially large, thus requiring relatively bulky molecules for efficient inhibition. To date, these BACE inhibitors have been limited by their toxicity and/or their exclusion from the CNS.

Another APP secretase proteinase, $\alpha$-secretase, is comprised of cell-surface metalloproteinases, a disintegrin and metalloproteinase-10 (ADAM-10) and -17 (22). These proteases cleave several known important substrates in addition to APP, including proTNF- $\alpha$ and pro-TGF- $\alpha$. The APP derivatives generated by $\alpha$-secretase are the released (or shed) ectodomain fragment $\mathrm{SAPP}_{\alpha}$ and the protein phosphatases 1 and 2A (PP1, PP2A) (ref. 23). As a result, when signals that involve activation of PKC or MAPK/ERK or inactivation of PP1, PP2A, or ROCK are transduced, APP metabolism is dramatically shifted toward the $\alpha$-secretase-mediated pathway and away from the $\beta$-secretase-mediated pathway and $A \beta$ generation (26). This phenomenon, known as regulated cleavage or regulated ectodomain shedding, appears to be due, at least in part, to redistribution of APP away from intracellular compartments and outward toward the plasma membrane (PM) where APP can encounter $\alpha$-secretase. $\alpha$-secretase per se may also be redistributed to the PM. Statins may lower the concentration of $A \beta$ by stimulating $\alpha$-secretase to yield a processing pattern that is indistinguishable from regulated cleavage modulated by PKC and MAPK/ERK (27). Recent evidence from Postina and colleagues using transgenic mice validates $\alpha$-secretase activation as a viable therapeutic opportunity that can antagonize brain $A \beta$ accumulation (28). These investigators showed that upregulation and downregulation of $\alpha$-secretase activity can modulate amyloid burden in plaque-forming mice in the predicted, bidirectional manner.

\section{What is the role of $A \beta$ in common forms of AD?}

Until 2004, one could justify the formulation that all autosomal dominant forms of $\mathrm{AD}$ could be linked to the accumulation of $\mathrm{A} \beta$ oligomers. A serious challenge to that model has arisen with the discovery of a family of individuals with a PS mutation manifesting clinically as frontotemporal dementia (FTD) and pathologically as "pure" NFT disease, i.e., there is no parenchymal or cerebrovascular amyloidosis in FTD (29). FTD is usually due to mutations involving the cytoskeletal protein tau, and these diseases are therefore called tauopathies (30). Since FTDs are NFT diseases, their existence has provided the strongest evidence that tangles cannot cause amyloidosis. No pathogenic PS1 mutation has heretofore been described as associating with pure NFT pathology. Furthermore, no generation of excess levels of A $\beta 42$ was observed when the FTD mutant PS was transfected into cultured cells (29). The possibility remains that the PS1 mutation is acting by elevating the levels of toxic A $\beta$ oligomers that induce tangle formation but not plaques, but no precedent exists for such a pathomechanism. Interest is now focused on the overexpression of this FTD mutant PS1 in transgenic mice, in order to assess further the nature of its pathogenicity.

The vast majority of all AD lacks a predictable, autosomal dominant mode of inheritance. While APP and the PSs consti- 

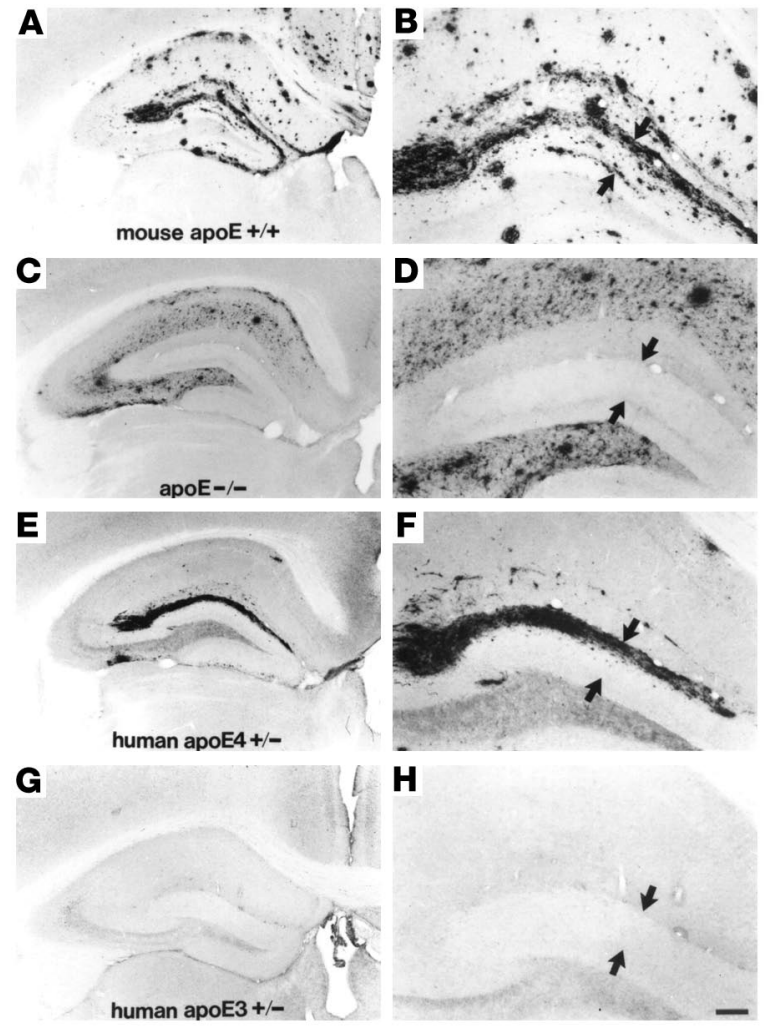

H

\section{Figure 7}

$A P O E$ isoform-specific regulation of $A \beta$ plaque burden. $A \beta$ plaque load is highest in the hippocampi of mice expressing murine apoE (A, magnified in $\mathbf{B}$ ) and lowest in $A P O E$ knockout animals ( $\mathbf{C}$, magnified in $\mathbf{D})$. Plaque load is moderate in the hippocampi of human $A P O E$ \&4-expressing mice ( $\mathbf{E}$, magnified in $\mathbf{F}$ ) as compared to murine apoE-expressing mice. Plaque load in the hippocampi of human $A P O E$ \&4-expressing mice is greater than plaque load in the hippocampi from human $A P O E$ $\varepsilon 3-$ expressing mice ( $\mathbf{G}$, magnified in $\mathbf{H}$ ). Amyloid deposits in the dentate gyrus, indicated by arrows, never develop in the absence of apoE. Scale bar: $150 \mu \mathrm{m}(\mathbf{A}, \mathbf{C}, \mathbf{E}$, and G) and $60 \mu \mathrm{m}$ (B, D, F, and H). Figure modified with permission from Proceedings of the National Academy of Sciences of the United States of America (40).

More recent studies, however, have raised questions about the relevance of these SDS-resistant apoE:A $\beta$ complexes under physiological conditions since immunoprecipitation assays indicate that apoE $\varepsilon 3$ and apoE $\varepsilon 4$ bind (36) $A \beta$ and stimulate fibrillogenesis (38) equivalently, at least under the conditions studied. This is bolstered by data obtained from purification and characterization of apoE:A $\beta$ complexes from the brains of subjects with various $A P O E$ genotypes, in which we demonstrated no effect of $A P O E$ genotype on the quality or composition of the apoE:A $\beta$ complex (39).

The best evidence linking APOE genotype to $\mathrm{A} \beta$ accumulation comes from studies of human $A P O E$ isoform-specific transgenic mice created on an $A P O E$ null background and crossed with mice bearing a pathogenic mutant form of APP (40). Plaque load was clearly enhanced in $\varepsilon 4$ mice as compared with $\varepsilon 3$ mice (ref. 40 ; Figure 7 ). We recently demonstrated that, unlike human $\varepsilon 3$, human $\varepsilon 4$ is associated with elevated levels of endogenous murine brain $A \beta$ as a function of aging and gender, even in mice that will therefore never develop cerebral amyloidosis. These data support the conclusion that there exists an effect of $\varepsilon 4$ on $A \beta$ upstream of fibrillogenesis (41).

\section{Are there other plausible hypotheses for the pathomechanisms of APOE polymorphisms?}

Another model that could explain, at least in part, the effects of $\varepsilon 4$ involves the relative deficiency of $\varepsilon 4$ as an antioxidant, since $\varepsilon 4$ lacks the cysteine residues that are present in $\varepsilon 2$ and $\varepsilon 3$ and help buffer oxidant stress, when $\varepsilon 4$ is compared with $\varepsilon 3$ or $\varepsilon 2$ (42). This dovetails with evidence that antioxidants can slow (43) the progression of $\mathrm{AD}$ and perhaps even prevent familial AD due to APP mutations (see above discussion about APOE $\varepsilon 2$ and APP mutations; ref. 18).

We included an assessment of this possibility in our evaluation of the effect of aging and $A P O E$ genotype on levels of nonamyloidogenic endogenous mouse $A \beta$ (41), extending the growing body of evidence indicating that oxidized prostaglandins known as isoprostanes might be useful markers for $\mathrm{AD}$ (reviewed in ref. 44). $\mathrm{F}_{2 \alpha}$-isoprostanes have been reported to rise in the tissues and/or body fluids of patients with $\mathrm{AD}$ and of transgenic plaque-forming mice at around the age of incipient amyloidosis (44-46). $F_{2 \alpha}$-isoprostanes have also been shown to stimulate both generation (47) and aggregation (48) of $A \beta$, possibly placing isoprostane accumulation upstream of $A \beta$ aggregation. This is an important point because human (but not murine) $A \beta$ is reported to be a pro-oxidant (49). We were able to dissociate the isoprostane elevation from the formation of histological amyloid by using mice bearing only endogenous murine APP and $\mathrm{A} \beta$ (41). Thus, plaque formation is not a prerequisite for elevation of brain isoprostane levels. Indeed, one model that we propose (41) suggests that oxidized lipids might sometimes initiate $\mathrm{A} \beta$ aggregation. 

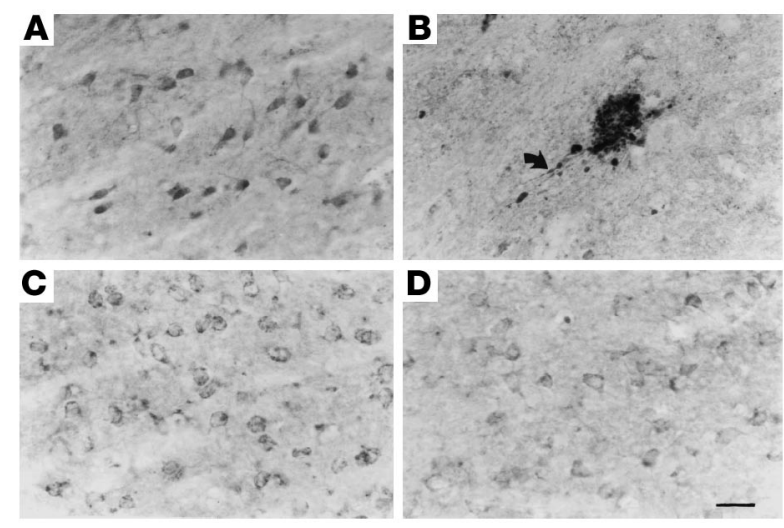

Figure 8

Comparison of the immunocytochemical distributions of APP (A and $\mathbf{B})$ and PS1 (C and $\mathbf{D})$ in control ( $\mathbf{A}$ and $\mathbf{C}$ ) and thiamine-deficient (B and D) mouse brain. Note the accumulation of APP immunoreactivity in a dystrophic neuritic cluster during thiamine deficiency (B). Arrow shows APP accumulation in an abnormal neurite arising from the neuritic cluster. PS1-immunostained sections adjacent to $\mathbf{A}$ and $\mathbf{B}$ reveal no accumulation of PS1 immunoreactivity in abnormal structures during thiamine deficiency except in areas of severe cell loss, in which the immunostaining is pale (D). Scale bar: $25 \mu \mathrm{m}$. Figure reproduced with permission from American Journal of Pathology (50).

Abnormal oxidation is one of the most consistent themes in all aging-related diseases of all organ systems, and it is interesting to note that the brain lesions resulting from oxidative toxicity induced by thiamine deficiency include abnormal, amyloid precursor-containing neuritic lesions (Figure 8; ref. 50). In ongoing studies, these lesions are now being induced in plaque-competent transgenic mice in order to determine whether the neuritic brain lesions induced by the oxidative reactions of thiamine deficiency accelerate amyloid deposition.

\section{What is the role of cholesterol in AD?}

The involvement of APOE polymorphisms in AD immediately raised the question of whether cholesterol might play a role in the risk for the disease. In evaluating this possibility, Sparks and colleagues determined that Watanabe rabbits on a $2 \%$ cholesterol diet developed increased levels of $\mathrm{A} \beta$ immunoreactivity in vesicles inside the neurons of

\section{Figure 9}

Possible A $\beta$-dependent and $A \beta$-independent mechanisms of neurotoxicity. In this model, 1 route toward neurotoxicity begins with either APP mutations or PS1 mutations (top center of figure). The pathway toward toxicity flows downward via elevated $A \beta 42$ levels and elevated oligomer levels. An alternative pathway is shown on the right side of the figure, whereby superoxide $\left(\mathrm{O}_{2}{ }^{\cdot-}\right)$ or hydrogen peroxide $\left(\mathrm{H}_{2} \mathrm{O}_{2}\right)$ oxidize lipids such as prostaglandins, forming $\mathrm{F}_{2 \alpha}$-isoprostanes. Both $\mathrm{H}_{2} \mathrm{O}_{2}$ and $\mathrm{F}_{2 \alpha}$-isoprostanes are known to accelerate $A \beta$ aggregation and presumably its oligomerization. This author proposes that toxicity in this pathway occurs both directly from reactive oxygen species $\left(\mathrm{H}_{2} \mathrm{O}_{2}\right.$ and $\left.\mathrm{O}_{2}{ }^{--}\right)$and via acceleration of $\mathrm{A} \beta$ oligomerization by these reactive oxygen species. their brains (51). Elucidation of the mechanism underlying this observation began to be vigorously pursued in plaque-forming transgenic mouse models, and soon several groups demonstrated that fat feeding increases brain plaque load and that hypocholesterolemic agents such as statins lower plaque burden $(52,53)$. In cultured neurons, both simvastatin and atorvastatin as well as cyclodextrin, a compound used in the laboratory to deplete cellular cholesterol, were shown to lower $\mathrm{A} \beta$ generation while activating the alternate, nonamyloidogenic $\alpha$-secretase pathway for APP metabolism $(27,54)$. At least some of the actions of statins on $\alpha$-secretase appear to occur via the rho/ROCK pathway, which can modulate $\mathrm{SAPP}_{\alpha}$ generation (25).

The epidemiological evidence linking cholesterol and AD is confusing. Some studies have concluded that statin use lowers the risk for dementia (55), while others conclude that this association is artifactual (56). Several epidemiological studies also show that lipid levels are lower in persons who develop AD, not higher (57). Perhaps the most encouraging evidence, albeit preliminary, is the apparent ability of atorvastatin to slow progression of dementia in AD (58). The publication of this report and the results of a large National Institute on Aging-sponsored prevention trial of statins are eagerly anticipated. At the moment, caution is recommended, especially when one reflects upon the ongoing efforts to reconcile epidemiological evidence that hormone replacement therapy (HRT) could delay or prevent $\mathrm{AD}$ with the disappointment of clinical trials of HRT. A primary prevention trial of HRT is now underway, focusing on institution of replacement during the perimenopausal period rather than the typical, late postmenopausal protocol.

\section{What antiamyloid medicines are in current clinical trials?}

Antiamyloid strategies fall into 3 basic categories: immunotherapy agents, antiaggregants, and secretase modulators. Active immunization (vaccination) has been limited by the side effect of

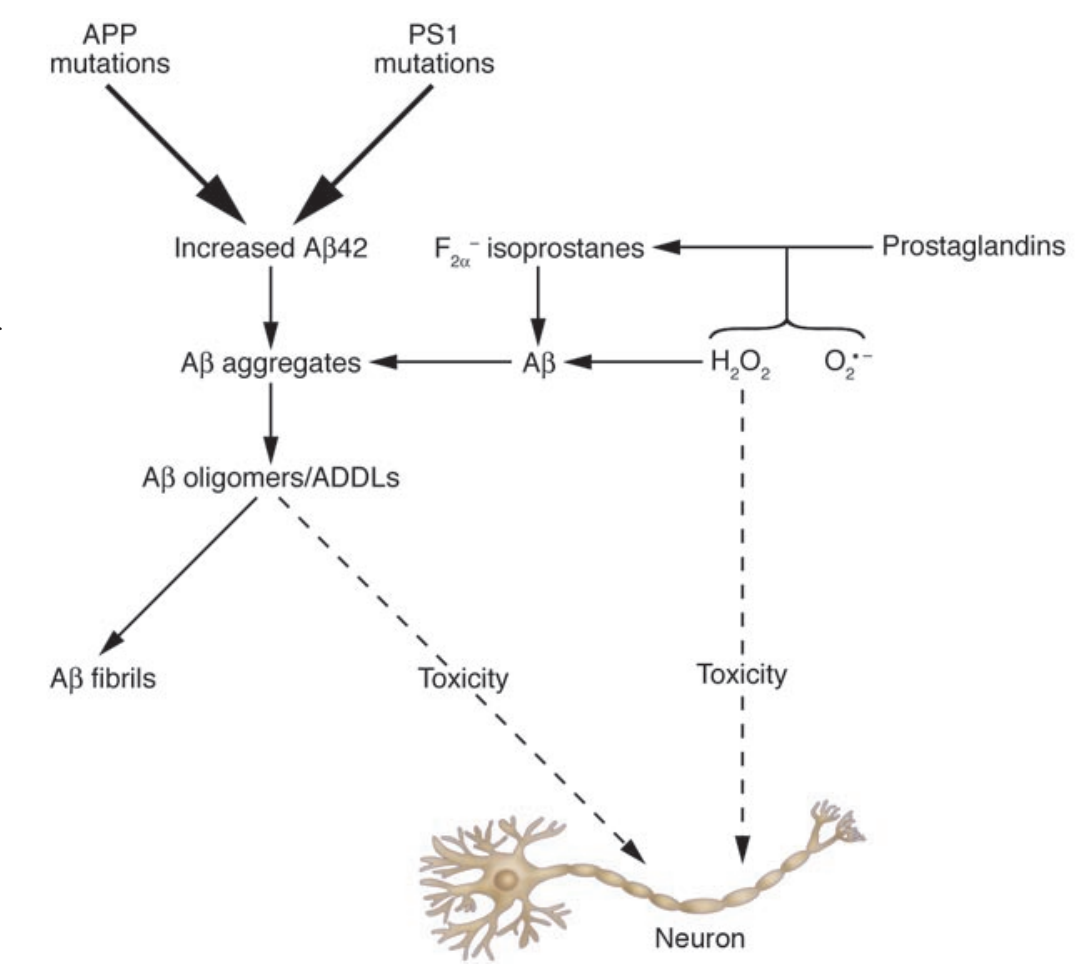


acute allergic encephalitis in 5\% of 300 subjects (59), but phase I trials of passive immunization are now underway. The antiaggregant approach is represented by commercial efforts at blocking the promotion of amyloidogenesis by glycosaminoglycans of the extracellular matrix. Glycosaminoglycan mimetics are currently in phases II and III of clinical trials aiming to evaluate their effectiveness at either slowing the progression of $\mathrm{AD}$ or preventing rebleeding due to cerebral amyloid angiopathy. Statins, as mentioned above, represent the best examples of $\alpha$-secretase activators that are currently in treatment and prevention trials. Flurbiprofen, a derivative of ibuprofen, is a modulator of $A \beta 42$ generation that is believed to allosterically modify $\gamma$-secretase so that generation of $A \beta 42$ is inhibited while an otherwise trace species, $A \beta 38$, is generated (60). A large $\mathrm{AD}$ prevention trial of conventional NSAIDs was aborted in 2005 when excess cardiac mortality became associated with their use. Other $\gamma$-secretase modulators are in the pipeline at several pharmaceutical companies, but progress is especially slow with such compounds because of the known risk factor of mechanism-based toxicity due to inhibition of Notch processing (61). The website www.clinicaltrials.gov remains the best source of up-to-date information on which trials are enrolling and whom to contact locally for further information.

\section{What is the role of $A \beta$ accumulation in common forms of AD?}

Genetic evidence indicates that accumulation of $A \beta$ in some biophysical form is injurious to the brain. However, this formulation is most defensible in the approximately 50 families worldwide who have early onset AD due to APP mutations. Also, data from studies of other cerebral amyloidoses (e.g., FBD and FDD) support a key role for amyloidosis in causing neurodegeneration. Metabolism and trafficking of APP are tightly controlled events, and it is entirely possible that excess $A \beta 42$ generation and/or accumulation is an initial symptom of some other primary problem (such as excess oxidation) that is itself independently neurotoxic. In such a scenario, however, it remains possible (and likely) that $\mathrm{A} \beta$ accumulation is a second, parallel pathway that is also toxic (Figure 9). This suggests that antiamyloid therapeutic strategies are likely to be beneficial in the treatment of $\mathrm{AD}$ even if they are not curative. Dissection of the genesis of the $A \beta$ accumulation phenotype is important in order to point the way to any upstream, primary lesions. The successful development of effective antiamyloid therapies - immunomodulators, antiaggregants, secretase modulators, or some combination of these - remains a key goal, the attainment of which is required in order to elucidate the role played by cerebral accumulation of toxic $\mathrm{A} \beta$ oligomers in the clinical picture of $\mathrm{AD}$.

\section{Acknowledgments}

The author acknowledges support from United States Public Health Service grants P01 AG10491 and R01 NS41017. In the past, the author has also received consultant fees and/or grant support from Parke-Davis, Wyeth, F. Hoffman-LaRoche Ltd., Pfizer Inc., Eisai Inc., and Andrx Corp.

Address correspondence to: Sam Gandy, Farber Institute for Neurosciences, Thomas Jefferson University, 900 Walnut Street, Suite 467, Philadelphia, Pennsylvania 19107, USA. Phone: (215) 5034200; Fax: (215) 503-4358; E-mail: samgandy@earthlink.net.
1. Mitchell, T.W., et al. 2002. Parahippocampal tau pathology in healthy aging, mild cognitive impairment, and early Alzheimer's disease. Ann. Neurol. 51:182-189.

2. Alford, M.F., Masliah, E., Hansen, L.A., and Terry, R.D. 1994. A simple dot-immunobinding assay for quantification of synaptophysin-like immunoreactivity in human brain. J. Histochem. Cytochem. 42:283-287.

3. Virchow, R. 1854. Zur cellulosefrage. Virchows Arch. Pathol. Anat. Physiol. 6:416-426.

4. Glenner, G.G., and Terry, W.D. 1974. Characterization of amyloid. Annu. Rev. Med. 25:131-135.

5. Glenner, G.G., and Wong, C.W. 1984. Alzheimer's disease and Down's syndrome: sharing of a unique cerebrovascular amyloid fibril protein. Biochem. Biophys. Res. Commun. 122:1131-1135.

6. Naslund, J., et al. 1994. Relative abundance of Alzheimer A beta amyloid peptide variants in Alzheimer disease and normal aging. Proc. Natl. Acad. Sci. U. S. A. 91:8378-8382.

7. Younkin, S.G. 1995. Evidence that A beta 42 is the real culprit in Alzheimer's disease. Ann. Neurol. 37:287-288.

8. Klein, W.L., Krafft, G.A., and Finch, C.E. 2001. Targeting small Abeta oligomers: the solution to an Alzheimer's disease conundrum? Trends Neurosci. 24:219-224

9. Kayed, R, et al. 2004. Permeabilization of lipid bilayers is a common conformation-dependent activity of soluble amyloid oligomers in protein misfolding diseases. J. Biol. Chem. 279:46363-46366.

10. Harper, J.D., and Lansbury, P.T., Jr. 1997. Models of amyloid seeding in Alzheimer's disease and scrapie: mechanistic truths and physiological consequences of the time-dependent solubility of amyloid proteins. Annu. Rev. Biochem. 66:385-407.

11. Naslund, J., et al. 2000. Correlation between elevated levels of amyloid beta-peptide in the brain and cognitive decline. JAMA. 83:1571-1577.

12. Hsiao, K., et al. 1996. Correlative memory deficits, Abeta elevation, and amyloid plaques in transgenic mice. Science. 274:99-102.

13. Gong, Y., et al. 2003. Alzheimer's disease-affected brain: presence of oligomeric A beta ligands (ADDLs) suggests a molecular basis for reversible memory loss. Proc. Natl. Acad. Sci. U. S. A. 100:10417-10422.

14. Georganopoulou, D.G., et al. 2005. Nanoparticlebased detection in cerebral spinal fluid of a soluble pathogenic biomarker for Alzheimer's disease. Proc. Natl. Acad. Sci. U. S. A. 102:2273-2276.

15. Gandy, S. 1999. Neurohormonal signaling pathways and the regulation of Alzheimer beta-amyloid precursor metabolism. Trends Endocrinol Metab. 10:273-279.

16. Nilsberth, C., et al. 2001. The 'Arctic' APP mutation (E693G) causes Alzheimer's disease by enhanced Abeta protofibril formation. Nat. Neurosci. 4:887-893.

17. Paivio, A., Jarvet, J., Graslund, A., Lannfelt, L., and Westlind-Danielsson, A. 2004. Unique physicochemical profile of beta-amyloid peptide variant Abeta140E22 G protofibrils: conceivable neuropathogen in Arctic mutant carriers. J. Mol. Biol. 339:145-159.

18. St. George-Hyslop, P., et al. 1994. Alzheimer's disease and possible gene interaction [letter]. Science. 263:537.

19. Ghiso, J., and Frangione, B. 2002. Amyloidosis and Alzheimer's disease. Adv. Drug Deliv. Rev. 54:1539-1551.

20. Carter, T.L., et al. 2004. Alzheimer amyloid precursor aspartyl proteinase activity in CHAPSO homogenates of Spodoptera frugiperda cells. Alzheimer Dis. Assoc. Disord. 18:261-263.

21. Vassar, R., et al. 1999. Beta-secretase cleavage of Alzheimer's amyloid precursor protein by the transmembrane aspartic protease BACE. Science.
286:735-741

22. Buxbaum, J.D., et al. 1998. Evidence that tumor necrosis factor alpha converting enzyme is involved in regulated alpha-secretase cleavage of the Alzheimer amyloid protein precursor. J. Biol. Chem. 273:27765-27767.

23. Caporaso, G.L., Gandy, S.E., Buxbaum, J.D., Ramabhadran, T.V., and Greengard, P. 1992. Protein phosphorylation regulates secretion of Alzheimer beta/A4 amyloid precursor protein. Proc. Natl. Acad. Sci. U. S. A. 89:3055-3059

24. Mills, J., et al. 1997. Regulation of amyloid precursor protein catabolism involves the mitogen-activated protein kinase signal transduction pathway. J. Neurosci. 17:9415-9422.

25. Pedrini, S., et al. 2005. Modulation of statin-activated shedding of Alzheimer APP ectodomain by ROCK. PLoS Med. 2:e18. doi:10.1371/journal. pmed.0020018.

26. Buxbaum, J.D., Koo, E.H., and Greengard, P. 1993. Protein phosphorylation inhibits production of Alzheimer amyloid beta/A4 peptide. Proc. Natl. Acad. Sci. U. S. A. 90:9195-9198.

27. Kojro, E., Gimpl, G., Lammich, S., Marz, W., and Fahrenholz, F. 2001. Low cholesterol stimulates the nonamyloidogenic pathway by its effect on the alpha-secretase ADAM 10. Proc. Natl. Acad. Sci. U. S. A. 98:5815-5820.

28. Postina, R., et al. 2004. A disintegrin-metalloproteinase prevents amyloid plaque formation and hippocampal defects in an Alzheimer disease mouse model. J. Clin. Invest. 113:1456-1464. doi:10.1172/JCI200420864.

29. Dermaut, B., et al. 2004. A novel presenilin 1 mutation associated with Pick's disease but not $\beta$-amyloid plaques. Ann. Neurol. 55:617-626.

30. Hutton, M. 2004. Presenilin mutations associated with fronto-temporal dementia [review]. Ann. Neurol. 55:604-606. 
31. Mayeux, R., et al. 1993. The apolipoprotein epsilon 4 allele in patients with Alzheimer's disease. Ann. Neurol. 34:752-754.

32. Farrer, L.A., et al. 1997. Effects of age, sex, and ethnicity on the association between apolipoprotein $\mathrm{E}$ genotype and Alzheimer disease. A meta-analysis. APOE and Alzheimer Disease Meta Analysis Consortium. JAMA. 278:1349-1356.

33. Martins, R.N., et al. 1995. ApoE genotypes in Australia: roles in early and late onset Alzheimer's disease and Down's syndrome. Neuroreport. 6:1513-1516.

34. LaDu, M.J., et al. 1994. Isoform-specific binding of apolipoprotein E to beta-amyloid. J. Biol. Chem. 269:23403-23406.

35. Zhou, Z., Smith, J.D., Greengard, P., and Gandy, S. 1996. Alzheimer amyloid-beta peptide forms denaturant-resistant complex with type epsilon 3 but not type epsilon 4 isoform of native apolipoprotein $\mathrm{E}$. Mol. Med. 2:175-180.

36. Zhou, Z., Relkin, N., Ghiso, J., Smith, J.D., and Gandy, S. 2002. Human cerebrospinal fluid apolipoprotein $\mathrm{E}$ isoforms are apparently inefficient at complexing with synthetic Alzheimer's amyloid-[beta] peptide (A[beta] 1-40) in vitro. Mol. Med. 8:376-381.

37. Yang, D.S., Smith, J.D., Zhou, Z., Gandy, S.E., and Martins, R.N. 1997. Characterization of the binding of amyloid-beta peptide to cell culture-derived native apolipoprotein E2, E3, and E4 isoforms and to isoforms from human plasma. J. Neurochem. 68:721-725.

38. Sweeney, D., Martins, R., LeVine, H., 3rd, Smith, J.D., and Gandy, S. 2004. Similar promotion of Abeta1-42 fibrillogenesis by native apolipoprotein E epsilon 3 and epsilon 4 isoforms. J. Neuroinflammation. 1:15-20.

39. Naslund, J., et al. 1995. Characterization of stable complexes involving apolipoprotein $\mathrm{E}$ and the amyloid beta peptide in Alzheimer's disease brain. Neuron. 15:219-228.

40. Holtzman, D.M., et al. 2000. Apolipoprotein E isoform-dependent amyloid deposition and neu- ritic degeneration in a mouse model of Alzheimer's disease. Proc. Natl. Acad. Sci. U. S. A. 97:2892-2897.

41. Yao, J., et al. 2004. Aging, gender and APOE isotype modulate Alzheimer's $A \beta$ peptides and $\mathrm{F}_{2}$-isoprostanes in the absence of detectable amyloid deposits. J. Neurochem. 90:1011-1018.

42. Miyata, M., and Smith, J.D. 1996. Apolipoprotein E allele-specific antioxidant activity and effects on cytotoxicity by oxidative insults and beta-amyloid peptides. Nat. Genet. 14:55-61.

43. Mayeux, R., and Sano, M. 1999. Treatment of Alzheimer's disease. N. Engl. J. Med. 341:1670-1679.

44. Pratico, D., and Sung, S. 2004. Lipid peroxidation and oxidative imbalance: early functional events in Alzheimer's disease. J. Alzheimers. Dis. 6:171-175.

45. Pratico, D., Uryu, K., Leight, S., Trojanoswki, J.Q., and Lee, V.M. 2001. Increased lipid peroxidation precedes amyloid plaque formation in an animal model of Alzheimer amyloidosis. J. Neurosci. 21:4183-4187.

46. Yao, Y., et al. 2004. Brain inflammation and oxidative stress in a transgenic mouse model of Alzheimer-like brain amyloidosis [serial online]. J. Neuroin flammation. 1:21. http://www.jneuroinflammation. com/content $/ 1 / 1 / 21$.

47. Qin, W., et al. 2003. Cyclooxygenase (COX)-1 and COX-2 potentiate beta-amyloid peptide generation through mechanisms that involve gamma-secretase activity. J. Biol. Chem. 278:50970-50977.

48. Boutoud, O., et al. 2002. Prostaglandin H2 (PGH2) accelerates formation of amyloid beta 1-42 oligomers. J. Neurochem. 82:1003-1006.

49. Huang, X., et al. 1999. The A beta peptide of Alzheimer's disease directly produces hydrogen peroxide through metal ion reduction. Biochemistry. 38:7609-7616

50. Calingasan, N.Y., et al. 1996. Novel neuritic clusters with accumulations of amyloid precursor protein and amyloid precursor-like protein 2 immunoreactivity in brain regions damaged by thiamine deficiency. Am. J. Pathol. 149:1063-1071.

51. Sparks, D.L., et al. 1994. Induction of Alzheimer- like beta-amyloid immunoreactivity in the brains of rabbits with dietary cholesterol. Exp. Neurol. 126:88-94.

52. Refolo, L.M., et al. 2000. Hypercholesterolemia accelerates the Alzheimer's amyloid pathology in a transgenic mouse model. Neurobiol. Dis. 7:321-331.

53. Refolo, L.M., et al. 2001. A cholesterol-lowering drug reduces beta-amyloid pathology in a transgenic mouse model of Alzheimer's disease. Neurobiol Dis. 8:890-899.

54. Simons, M., et al. 1998. Cholesterol depletion inhibits the generation of beta-amyloid in hippocampal neurons. Proc. Natl. Acad. Sci. U. S. A. 95:6460-6464.

55. Wolozin, B., Kellman, W., Ruosseau, P., Celesia, G.G., and Siegel, G. 2000. Decreased prevalence of Alzheimer disease associated with 3-hydroxy3-methyglutaryl coenzyme A reductase inhibitors. Arch. Neurol. 57:1439-1443.

56. Li, G., et al. 2004. Statin therapy and risk of dementia in the elderly: a community-based prospective cohort study. Neurology. 63:1624-1628.

57. Romas, S.N., Tang, M.X., Berglund, L., and Mayeux, R. 1999. APOE genotype, plasma lipids, lipoproteins, and AD in community elderly. Neurology. 53:517-521.

58. Sparks, D.L., et al. 2004. Benefit of atorvastatin in the treatment of Alzheimer disease. Neurobiol. Aging. 25(Suppl. 1):S24.

59. Nicoll, J.A., et al. 2003. Neuropathology of human Alzheimer disease after immunization with amyloidbeta peptide: a case report. Nat. Med. 9:448-452.

60. Weggen, S., et al. 2001. A subset of NSAIDs lower amyloidogenic Abeta42 independently of cyclooxygenase activity. Nature. 414:212-216.

61. Fortini, M.E. 2002. Gamma-secretase-mediated proteolysis in cell-surface-receptor signalling. Nat. Rev. Mol. Cell Biol. 3:673-684.

62. Shoghi-Jadid, K., et al. 2002. Localization of neurofibrillary tangles and beta-amyloid plaques in the brains of living patients with Alzheimer disease. Am. J. Geriatr. Psychiatry. 10:24-35. 